Pacific Journal of Mathematics

MATRIX RINGS OF FINITE DEGREE OF NILPOTENCY 


\title{
MATRIX RINGS OF FINITE DEGREE OF NILPOTENCY
}

\author{
Abraham A. KLein
}

The degree of nilpotency of a ring $R$ is defined to be the supremum of the orders of nilpotency of its nilpotent elements and it is denoted by $\nu(R)$. We consider the degree of nilpotency of the ring of $m \times m$ matrices $R_{m}$ over a ring $R$. We obtain given results concerning the degrees $\nu\left(R_{m}\right)$ for distinct $m$ 's, in the case $R$ has no nonzero two-sided annihilators. It is shown that if $\nu\left(R_{m}\right)=m$ for some $m$, and if $R^{\prime}$ is a ring containing $R$ as an ideal such that $R^{\prime}$ has no nonzero two-sided annihilators of $R$, then $\nu\left(R_{m}^{\prime}\right)=m$. An application of this result is given.

$R$ will always be a nonzero associative ring. If $a \in R$ is nilpotent, we denote its order of nilpotency by $\nu(a)=\min \left\{k \mid a^{k}=0\right\}$, and if $a$ is not nilpotent we put: $\nu(a)=0$. The degree of nilpotency $\nu(R)$ of $R$ is defined by

$$
\nu(R)=\sup _{a \in R} \nu(a) .
$$

If $R$ is a ring without nonzero nilpotent elements then $\nu(R)=\nu(0)=$ 1 , and we shall soon see that the ring $R_{m}$ of $m \times m$ matrices over $R$ satisfies $\nu\left(R_{m}\right) \geqq m$ (Lemma 1 ).

There exist rings $R$ satisfying $\nu\left(R_{m}\right)>m$ and in [3] was shown that such an $R$ may even be a (noncommutative) integral domain. The object of this paper is to deal with rings $R$ which satisfy $\nu\left(R_{m}\right)=$ $m$ for some $m$. We denote this condition by $\mathfrak{N}_{m}$. First we shall consider the degree of nilpotency of matrix rings over rings without nonzero two-sided annihilators. Then we give some conditions equivalent to $\mathfrak{N}_{m}$. Our main result is: If a nonzero ideal in an integral domain $R$ satisfies $\mathfrak{R}_{m}$ then $R$ itself satisfies $\mathfrak{N}_{m}$. This implication resembles the following one: If a nonzero ideal in an integral domain $R$ is embeddable in a field then $R$ itself is embeddable in a field [1]. This result together with other results obtained in [4], lead us to the conjecture: "The conditions $\mathfrak{N}_{m}, m=1,2, \cdots$, are sufficient for embedding an integral domain in a field.

Our result is applied to prove that a ring which has no nonzero two-sided annihilators and satisfies $\mathfrak{N}_{m}$ is embeddable in a ring with an identity which satisfies $\mathfrak{R}_{m}$.

I wish to thank G. M. Bergman for his suggestions and comments on this paper.

2. Rings without nonzero two-sided annihilators. The following notations will be used later. 
If $a \in R$ then we denote by $a E i j$ the matrix with $a$ in its $(i, j)$ position and 0 elsewhere.

If $A=\left(a_{i j}\right) \in R_{m}$ and $r$ is an integer $\geqq 1$, we denote the $(i, j)$ entry of $A^{r}$ by $a_{i j}^{(r)}$. Since $A^{r} A^{s}=A^{r+s}$ we have:

$$
\sum_{k=1}^{m} a_{i k}^{(r)} a_{k j}^{(s)}=a_{i j}^{(r+s)} \text {. }
$$

LEMMA 1 . If $R$ is not nilpotent then $\nu\left(R_{m}\right) \geqq m$ for each $m \geqq 1$.

Proof. The result is trivial for $m=1$, so let $m \geqq 2$. Since $R^{m-1} \neq$ 0 , there exist $a_{1}, \cdots, a_{m-1} \in R$ such that $a_{1} \cdots a_{m-1} \neq 0$. Hence the matrix $A=\sum_{i=1}^{m-1} a_{i} E_{i, i+1}$ satisfies $A^{m-1}=a_{1} \cdots a_{m-1} E_{1 m} \neq 0$ and $A^{m}=$ 0 . Thus, $\nu\left(R_{m}\right) \geqq \nu(A)=m$.

COROLLARY. For rings $R$ without nonzero nilpotent elements, the condition $\mathfrak{N}_{m}$ is inherited by (nonzero) subrings.

Indeed, if $R^{\prime}$ is a subring of $R$ then $\nu\left(R_{m}^{\prime}\right) \geqq m$ since $R^{\prime}$ is not nilpotent. If $R$ satisfies $\mathfrak{N}_{m}$ then since $R_{m}^{\prime}$ is a subring of $R_{m}$ we have $\nu\left(R_{m}^{\prime}\right) \leqq \nu\left(R_{m}\right)=m$.

If $S$ is a nonempty subset of $R$, we denote its right (left) annihilator in $R$ by $r_{R}(S)\left(l_{R}(S)\right)$. Clearly $r_{R}(S) \cap l_{R}(S)$ is the set of twosided annihilators of $S$ in $R$.

Note that if $R$ is a (nonzero) ring such that $r_{R}(R) \cap l_{R}(R)=\{0\}$ then $R$ is not nilpotent.

The proof of our next result is similar to that of [4, Lemma 9].

Lemma 2. If $r_{R}(R) \cap l_{R}(R)=\{0\}$ and $A \in R_{m}$ is nilpotent of order $h$, then there exist a matrix $B \in R_{m+1}$ which is nilpotent of order $h+1$.

Proof. If $h=1$ then $A=0$ and the result is trivial. If $h \geqq 2$ then $A^{h-1} \neq 0$ and there exist $p$ and $q, 1 \leqq p, q$, $\leqq m$, such that $a_{p q}^{(h-1)} \neq$ 0 . Since $r_{R}(R) \cap l_{R}(R)=\{0\}$, there exists an element $b \in R$ such that either $b a_{p q}^{(h-1)} \neq 0$ or $a_{p q}^{(h-1)} b \neq 0$. Assume that we have $a_{p q}^{(h-1)} b \neq 0$ (the other case is treated similarly). Let $A_{1}$ be the matrix of $R_{m+1}$ obtained from $A$ by adjoining a row and a column of zeros and let $B=A_{1}+$ $b E_{q, m+1}$. The powers of $B$ are given by

$$
B^{k}=A_{1}^{k}+\sum_{i=1}^{m} \alpha_{i q}^{(k-1)} b E_{i, m+1}, k \geqq 2 .
$$

Since $A_{1}^{h}=0$ and $a_{p q}^{(h-1)} b \neq 0$ we obtain $B^{h} \neq 0$ and $B^{h+1}=0$.

This immediately yields:

THEOREM 3. Let $R$ be a ring such that $r_{R}(R) \cap l_{R}(R)=\{0\}$. If $\nu\left(R_{m}\right) \geqq h$ then $\nu\left(R_{m+r}\right) \geqq h+r$ for each $r \geqq 1$, and if $\nu\left(R_{m}\right) \leqq h$ then $\nu\left(R_{m-r}\right) \leqq h-r$ for each $r=1,2, \cdots, m-1$. 
THEOREM 4. If $r_{R}(R) \cap l_{R}(R)=\{0\}$ and $R$ satisfies $\mathfrak{N}_{m}$ for some $m$, then it also satisfies $\mathfrak{N}_{k}$ for $k=1,2, \cdots, m-1$. In particular it follows that $R$ has no nonzero nilpotent elements.

\section{Conditions equivalent to $\mathfrak{N}_{m}$.}

THEOREM 5. Let $m$ be a fixed integer $>1$. The following conditions are equivalent for rings $R$ without nonzero nilpotent elements.

(i) $\mathfrak{R}_{m}: \nu\left(R_{m}\right)=m$

(ii) For all $C \in R_{m}, C^{m+1}=0$ implies $C^{m}=0$.

(iii) For all $A, B \in R_{m},(A B)^{m}=0$ implies $(B A)^{m}=0$.

Proof. It is clear that (i) implies (ii). If (ii) holds and $(A B)^{m}=0$ then $(B A)^{m+1}=B(A B)^{m} A=0$, hence $(B A)^{m}=0$ and (iii) holds.

Assume (iii) holds and we proceed to prove (i). Since $R$ has no nonzero nilpotent elements $r_{R}(R)=l_{R}(R)=0$, so $\nu\left(R_{m}\right) \geqq m$. Let $C=$ $\left(c_{i j}\right) \in R_{m}$, we have to prove that $\nu(C) \leqq m$. Assume $\nu(C)=h>m$ and let $c_{p q}^{(h-1)} \neq 0$. We define two matrices $A=\left(a_{i j}\right) \in R_{m}$ and $B=\left(b_{i j}\right) \in R_{m}$ as follows:

$$
\begin{aligned}
& a_{i j}=\left\{\begin{array}{l}
c_{p j}^{(i)}, i=1, \cdots, m-1 \\
c_{p j}^{(h-1)}, i=m
\end{array},\right. \\
& j=1, \cdots, m \text {. } \\
& b_{i j}=c_{i q}^{(h-j)}, \\
& i, j=1, \cdots, m \text {. }
\end{aligned}
$$

Using (1) we obtain for $j=1, \cdots, m$

$$
\begin{aligned}
\sum_{k=1}^{m} a_{i k} b_{k j} & =c_{p q}^{(h+i-j)}, \\
\sum_{k=1}^{m} a_{m k} b_{k j} & =c_{p q}^{(h+h-1-j)} .
\end{aligned}
$$

Since $C^{h}=0$, it follows that $C^{h+r}=0$ and $c_{p q}^{(h+r)}=0$ for each $r \geqq 0$. Hence the $(i, j)$ entry of $A B$ is 0 for $i \geqq j$, and it is $c_{p q}^{(h-1)}$ for $j=i+$ $1, i=1, \cdots, m-1$. This implies that $(A B)^{m-1}=\left(c_{p q}^{(h-1)}\right)^{m-1} E_{1 m}$ and $(A B)^{m}=0$. Since (iii) holds we have $(B A)^{m}=0$. But

$$
(B A)^{m}=B(A B)^{m-1} A
$$

and its $(i, j)$ entry is $b_{i 1}\left(c_{p q}^{(h-1)}\right)^{m-1} a_{m j}=0$. Taking $i=p$ and $j=q$ we obtain $\left(c_{p q}^{(h-1)}\right)^{m+1}=0$ and since $R$ has no nonzero nilpotent elements, it follows that $c_{p q}^{(h-1)}=0$, a contradiction. Hence $h \leqq m$ and $R$ satisfies (i).

4. The main result. If $T \neq 0$ is an ideal in $R$ and $T$ as a ring satisfies $\mathfrak{N}_{m}$, then it does not follow that $R$ satisfies $\mathfrak{N}_{m}$, even if $R$ has no nonzero nilpotent elements. Indeed, $R$ may be a direct sum of $T$ and a ring $R^{\prime}$ such that $\nu\left(R_{m}^{\prime}\right)>m$ and it is possible to choose 
$T$ and $R^{\prime}$ without nonzero nilpotent elements. Clearly, here the twosided annihilator of $T$ in $R$ is not 0 . On the other hand we have:

THEOREM 6. If $T$ is an ideal in $R$ such that $r_{R}(T) \cap l_{R}(T)=\{0\}$ and $\nu\left(T_{m}\right)=m$, then $\nu\left(R_{m}\right)=m$.

Proof. We have $\left.r_{T}(T) \cap l_{T}(T) \leqq r_{R}(T) \cap\right\urcorner_{R}(T)=0$ and $\nu\left(T_{m}\right)=m$, hence it follows by Theorem 4 that $T$ has no nonzero nilpotent elements. Since $R_{m}$ contains $T_{m}$ we have $\nu\left(R_{m}\right) \geqq m$. Let $C \in R_{m}$, we have to prove that $\nu(C) \leqq m$. As in the proof of Theorem 5 , assume $\nu(C)=$ $h>m$ and $c_{p q}^{(h-1)} \neq 0$. Construct the same matrices $A$ and $B$ and take arbitrary elements $a, b \in T$. Then $A_{1}=a A$ and $B_{1}=B b$ belong to $T_{m}$. We have $A_{1} B_{1}=a(A B) b$, hence the $(i, j)$ entry of $A_{1} B_{1}$ is 0 for $i \geqq j$ and it is $a c_{p q}^{(h-1)} b$ for $j=i+1, i=1, \cdots, m-1$. From this it follows that $\left(A_{1} B_{1}\right)^{m-1}=\left(\alpha c_{p q}^{(k-1)} b\right)^{m-1} E_{1 m}$ and $\left(A_{1} B_{1}\right)^{m}=0$. Since $A_{1}, B_{1} \in T_{m}$ and $\nu\left(T_{m}\right)=m$ it follows that $\left(B_{1} A_{1}\right)^{m}=0$. As in the proof of Theorem 5 we obtain that the $(p, q)$ entry of $B_{1}\left(A_{1} B_{1}\right)^{m-1} A_{1}=0$ is

$$
c_{p q}^{(h-1)} b\left(a c_{p q}^{(h-1)} b\right)^{m-1} a c_{p q}^{(h-1)}=0 \text {. }
$$

This implies that

$$
\left(b a c_{p q}^{(h-1)}\right)^{m+1}=0,\left(a c_{p q}^{(h-1)} b\right)^{m+1}=0,\left(c_{p q}^{(h-1)} b a\right)^{m+1}=0 .
$$

Since $T$ has no nonzero nilpotent elements it follows that

$$
b a_{p q}^{(h-1)}=0, a c_{p q}^{(h-1)} b=0, c_{p q}^{(h-1)} b a=0 .
$$

This is true for all $a, b \in T$, hence $a c_{p q}^{(h-1)} \in r_{T}(T) \cap l_{T}(T)=\{0\}$ and $c_{p q}^{(h-1)} b \in r_{T}(T) \cap l_{T}(T)=\{0\}$ and this implies that $c_{p q}^{(h-1)} \in r_{R}(T) \cap l_{R}(T)=$ $\{0\}$; a contradiction. Hence $h \leqq m$ and $\nu\left(R_{m}\right)=m$.

If $R$ is an integral domain and $T$ a nonzero ideal in $R$, then it is clear that $r_{R}(T)=l_{R}(T)=\{0\}$, hence we obtain our main result which is:

THeOREM 7. If $R$ is an integral domain and $T \neq 0$ an ideal in $R$ which satisfies $\mathfrak{I}_{m}$, then $R$ also satisfies $\mathfrak{N}_{m}$.

5. Embedding. Let $R$ be a ring without nonzero nilpotent elements. Embed $R$ in a ring $R^{\prime}$ with 1 in the usual way $[2$, p. 86]: $R^{\prime}=R+I, R \cap I=0$, where $I$ is the ring of integers. $R$ is an ideal in $R^{\prime}$ and since $r_{R}(R)=l_{R}(R)=\{0\}$ it follows that $r_{R^{\prime}}(R) \cap R=l_{R^{\prime}}(R) \cap$ $R=\{0\}$. Thus, $R$ is embeddable in $R^{\prime} / r_{R^{\prime}}(R)=R^{\prime \prime}$. One shows easily that $r_{R^{\prime}}(R)=l_{R^{\prime}}(R)$. If we identify $R$ with its image in $R^{\prime \prime}$ we obtain that $R$ is an ideal in $R^{\prime \prime}$ and $r_{R^{\prime \prime}}(R)=\{0\}$. Hence by Theorem 6 we obtain: 
THEOREM 8. If $R$ is a ring without nonzero nilpotent elements and satisfies $\mathfrak{N}_{m}$, then $R$ is embeddable in a ring with 1 which satisfies $\mathfrak{R}_{m}$.

If $R$ is an integral domain then the ring $R^{\prime \prime}$ obtained above is also an integral domain. Thus, we have:

COROLLARY. If $R$ is an integral domain which satisfies $\mathfrak{R}_{m}$ then $R$ is embeddable in an integral domain with 1 which satisfies $\mathfrak{R}_{m}$.

Note that this result enables us to simplify the proof in [4, Theorem 7] taking $t=1$.

Now, if $R$ is a ring with 1 and satisfies $\mathfrak{N}_{m}$ then $R$ has no nonzero nilpotent elements since $r_{R}(R)=\{0\}$. Let $C$ be the center of $R$ and assume that the nonzero elements of $C$ are regular in $R$. Thus, we may embed $R$ in the ring $R^{\prime}=\left\{a c^{-1} \mid a \in R, 0 \neq c \in C\right\}$ whose center is the quotient field of the commutative integral domain $C$. If $B=$ $\left(b_{i j}\right) \in R_{m}^{\prime}$ then it is possible to write its entries with a common denominator: $b_{i j}=a_{i j} c^{-1}, a_{i j} \in R, 0 \neq c \in C, 1 \leqq i, j \leqq m$. Let $A=\left(a_{i j}\right) \in$ $R_{m}$ then $B c=A$. If $B$ is nilpotent then $A$ is also nilpotent and since $R$ satisfies $\mathfrak{N}_{m}$ we have $A^{m}=0$. It follows that $B^{m} c^{m}=(B c)^{m}=0$ and so $B^{m}=0$ since $c^{m}$ is a unit in $R^{\prime}$. We have proved:

THeOREM 9. If $R$ is a ring with 1 which satisfies $\mathfrak{R}_{m}$ and all the elements of its center $C$ are regular, then $R$ is embeddable in a central K-algebra which satisfies $\mathfrak{N}_{m}, K$ the field of fractions of $C$.

\section{REFERENCES}

1. I. E. Burmistrovic. On the embedding of rings into fields, Sibirsk M. Z., 4 (1963), 1235-1240.

2. N. Jacobson, Lectures in Abstract Algebra I, Van Nostrand, 1951.

3. A. A. Klein, Rings nonembeddable in fields with multiplicative semigroups embeddable in groups, J. Algebra, 7 (1967), 100-125.

4. - Necessary conditions for embedding rings into fields, Trans. Amer. Math. Soc., 137 (1969), 141-151.

Received February 25, 1970.

TEL-AVIV UNIVERSITY

AND

YALE UNIVERSITY 



\section{PACIFIC JOURNAL OF MATHEMATICS}

\section{EDITORS}

H. SAMELson

Stanford University

Stanford, California 94305

R. R. PHelPS

University of Washington

Seattle, Washington 98105
J. Dugundu

Department of Mathematics

University of Southern Californle

Los Angeles, California 9.0007

RICHARD ARENS

University of California

Los Angeles, California 9.0024

\section{ASSOCIATE EDITORS}
E. F. BECKENBACH
B. H. NeumanN
F. WOLE
K. Yoshida

\section{SUPPORTING INSTITUTIONS}

UNIVERSITY OF BRITISH COLUMBIA

CALIFORNIA INSTITUTE OF TECHNOLOGY

UNIVERSITY OF CALIFORNIA

MONTANA STATE UNIVERSITY

UNIVERSITY OF NEVADA

NEW MEXICO STATE UNIVERSITY

OREGON STATE UNIVERSITY

UNIVERSITY OF OREGON

OSAKA UNIVERSITY

UNIVERSITY OF SOUTHERN CALIFORNIA

\author{
STANFORD UNIVERSITY \\ UNIVERSITY OF TOKYO \\ UNIVERSITY OF UTAH \\ WASHINGTON STATE UNIVERSITY \\ UNIVERSITY OF WASHINGTON \\ AMERICAN MATHEMATICAL SOCIETY \\ CHEVRON RESEARCH CORPORATION \\ NAVAL WEAPONS CENTER
}

The Supporting Institutions listed above contribute to the cost of publication of this Journal, but they are not owners or publishers and have no responsibility for its content or policies.

Mathematical papers intended for publication in the Pacific Journal of Mathematics should be in typed form or offset-reproduced, (not dittoed), double spaced with large margins. Underline Greek letters in red, German in green, and script in blue. The first paragraph or two must be capable of being used separately as a synopsis of the entire paper. The editorial "we" must not be used in the synopsis, and items of the bibliography should not be cited there unless absolutely necessary, in which case they must be identified by author and Journal, rather than by item number. Manuscripts, in duplicate if possible, may be sent to any one of the four editors. Please classify according to the scheme of Math. Rev. Index to Vol. 39. All other communications to the editors should be addressed to the managing editor, Richard Arens, University of California, Los Angeles, California, 90024.

50 reprints are provided free for each article; additional copies may be obtained at cost in multiples of 50 .

The Pacific Journal of Mathematics is published monthly. Effective with Volume 16 the price per volume (3 numbers) is $\$ 8.00$; single issues, $\$ 3.00$. Special price for current issues to individual faculty members of supporting institutions and to individual members of the American Mathematical Society: $\$ 4.00$ per volume; single issues $\$ 1.50$. Back numbers are available.

Subscriptions, orders for back numbers, and changes of address should be sent to Pacific Journal of Mathematics, 103 Highland Boulevard, Berkeley, California, 94708.

PUBLISHED BY PACIFIC JOURNAL OF MATHEMATICS, A NON-PROFIT CORPORATION

Printed at Kokusai Bunken Insatsusha (International Academic Printing Co., Ltd.), 7-17, Fujimi 2-chome, Chiyoda-ku, Tokyo, Japan. 


\section{Pacific Journal of Mathematics}

\section{Vol. 36, No. 2 December, 1971}

George E. Andrews, On a partition problem of H. L. Alder ............ 279

Thomas Craig Brown, An interesting combinatorial method in the theory of locally finite semigroups .......................... 285

Yuen-Kwok Chan, A constructive proof of Sard's theorem ............. 291

Charles Vernon Coffman, Spectral theory of monotone Hammerstein

operators...................................... 303

Edward Dewey Davis, Regular sequences and minimal bases .......... 323

Israel (Yitzchak) Nathan Herstein and Lance W. Small, Regular elements in

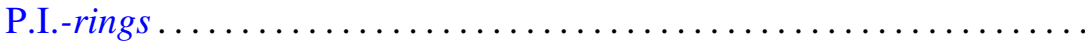

Marcel Herzog, Intersections of nilpotent Hall subgroups ..............

W. N. Hudson, Volterra transformations of the Wiener measure on the space

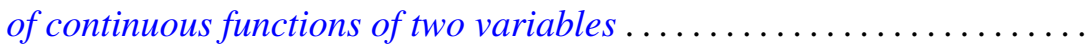

J. H. V. Hunt, An n-arc theorem for Peano spaces ................ 351

Arnold Joseph Insel, A decomposition theorem for topological group

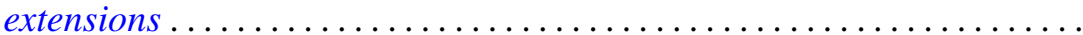

Caulton Lee Irwin, Inverting operators for singular boundary value

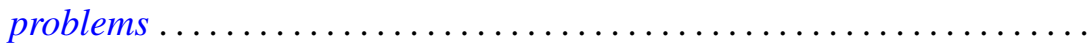

Abraham A. Klein, Matrix rings of finite degree of nilpotency ............ 387

Wei-Eihn Kuan, On the hyperplane section through a rational point of an algebraic variety...

John Hathway Lindsey, II, On a six-dimensional projective representation of $\mathrm{PSU}_{4}(3)$

Jorge Martinez, Approximation by archimedean lattice cones ...

J. F. McClendon, On stable fiber space obstructions .........

Mitsuru Nakai and Leo Sario, Behavior of Green lines at the Kuramochi boundary of a Riemann surface ....................

Donald Steven Passman, Linear identities in group rings. I. .

Donald Steven Passman, Linear identities in group rings. II ...

David S. Promislow, The Kakutani theorem for tensor products of

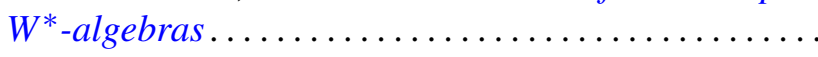

Richard Lewis Roth, On the conjugating representation of a finite group

Bert Alan Taylor, On weighted polynomial approximation of entire functions...

William Charles Waterhouse, Divisor classes in pseudo Galois

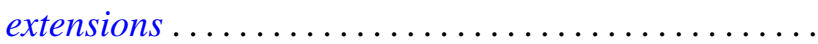

Chi Song Wong, Subadditive functions ...

Ta-Sun $\mathrm{Wu}$, A note on the minimality of certain bitransformation groups 